\title{
Biópsia de pele e seu estudo histológico. Por quê? Para quê? Como? Parte I*
}

\author{
Skin biopsy and its histopathologic analysis. Why? What for? How? Part I
}

\author{
Betina Werner ${ }^{1}$
}

Resumo: O estudo anatomopatológico é, na maioria das vezes, considerado padrão-ouro para esclarecimento diagnóstico de lesões neoplásicas ou inflamatórias de pele. Para que o resultado histopatológico da biópsia de pele seja satisfatório, no entanto, o médico deve estar ciente das razões para fazê-la em seguida de exame microscópico, dos objetivos a serem atingidos e da melhor maneira de se realizá-la, incluindo a escolha da melhor técnica e do melhor local anatômico para se retirar o fragmento de pele, dependendo da doença.

Palavras-chave: Biópsia; Microscopia; Pele; Pele/patologia

\begin{abstract}
Histopathologic study is considered gold-standard for diagnosis of skin lesions, either neoplastic or inflammatory, most of the times. To take the most out of a skin biopsy, physicians should be aware of the reasons for performing the procedure followed by microscopic examination, the objectives to be achieved and the best manner to do the biopsy, including the best technique and best anatomical site for a particular disease.

Keywords: Biopsy; Microscopy; Skin; Skin/pathology
\end{abstract}

\section{INTRODUÇÃO}

Ao contrário do que possa parecer, a biópsia com estudo anatomopatológico é processo complexo. A peculiaridade desse procedimento é o número de variáveis envolvidas para que o seu objetivo seja completamente atingido, ou seja, o diagnóstico correto e preciso. Desde o momento em que o dermatologista decide realizar uma biópsia de pele até aquele em que recebe a conclusão da análise microscópica, muitos fatores podem prejudicar o sucesso da biópsia de pele. Decisão quanto ao local anatômico e da lesão de que será colhido o fragmento de pele, escolha da técnica de biópsia, identificação, manuseio e fixação do espécime, preenchimento da requisição do exame anatomopatológico, análise macroscópica do fragmento de pele, processamento histológico e confecção das lâminas, estudo microscópico com formula- ção do diagnóstico, e interpretação do resultado do laudo anatomopatológico são etapas, todas muito importantes, que influenciam diretamente o resultado final.

Este artigo foi dividido em duas partes. A primeira tem o intuito de discutir o "porquê" de a biópsia de pele ser realizada com estudo anatomopatológico e seu "para que", ou seja, as razões que justificam o procedimento em si. Além disso, a primeira parte introduz o "como" fazer para uma biópsia de pele ser adequada, iniciando pela discussão da importância das informações clínicas que devem acompanhar o fragmento de pele enviado para análise. A segunda parte tem como objetivo completar a abordagem do "como", discutindo a decisão de qual técnica e a escolha de qual lesão e região da pele são mais apropria-

Recebido em 05.5.2009.

Aprovado pelo Conselho Editorial e aceito para publicação em 29.05.09.

* Trabalho realizado no Diagnose, serviço privado de patologia cirúrgica e dermatopatologia - Curitiba (PR), Brasil.

Conflito de interesse: Nenhum / Conflict of interest: None

Suporte financeiro: Nenhum / Financial funding: None

Especialista em dermatopatologia pela Sociedade Internacional de Dermatopatologia. Mestre em clínica cirúrgica, com área de concentração em patologia cirúrgica, pela Universidade Federal do Paraná. Doutora em saúde da criança e do adolescente, com área de concentração em dermatopatologia, pela Universidade Federal do Paraná. Professora/dermatopatologista voluntária/convidada dos Serviços de Anatomia Patológica e Dermatologia e da disciplina de anatomia patológica, Universidade Federal do Paraná (UFPR) - Curitiba (PR), Brasil. 
das para o estudo microscópico de algumas doenças específicas, além de tratar de algumas situações especiais, como a abordagem das biópsias de lesões melânicas e de couro cabeludo.

\section{POR QUÊ?}

Segundo um parecer de 1995 do Conselho Federal de Medicina (PC/CFM 44/95), "o exame anatomopatológico de fragmentos ou partes retirados do organismo humano deve ser feito consoante dever do médico de agir com o máximo zelo e o melhor de sua capacidade profissional, sempre em favor do paciente e sem caráter obrigatório". "Por outro lado, a Sociedade Brasileira de Patologia, no parecer 43/2006, manifesta-se da seguinte maneira: "o paciente é proprietário de suas células, tecidos e órgãos, que não podem ser simplesmente descartados em função da vontade médica. Torna-se necessário esclarecer o porquê da retirada de seus tecidos, bem como informá-lo dos objetivos de um procedimento anatomopatológico, cabendo ao paciente consentir ou não no desprezo de seu material". ${ }^{2} \mathrm{E}$ a mesma Sociedade reforça que "essa decisão deve ser documentada com a utilização de um Termo de Consentimento Esclarecido, sem o qual o médico assistente pode ser posteriormente responsabilizado, pelo paciente ou familiar, em caso de evolução insatisfatória ou inesperada da doença". ${ }^{2}$ Independentemente das opiniões desses órgãos regulamentadores, no fim das contas e na maioria das vezes, é o dermatologista, cirurgião dermatológico, cirurgião plástico ou outro médico especialista ou não que decidirá sozinho o que fazer com o fragmento de pele retirado do paciente. Doenças inflamatórias à parte, tumores e cistos cutâneos nem sempre são enviados para estudo anatomopatológico, dependendo do grau de certeza do clínico ou cirurgião sobre o caráter benigno da lesão. Em um país grande como o Brasil a formação da capacidade de diagnóstico clínico de lesões de pele por parte dos médicos é heterogênea, mesmo entre dermatologistas, especialmente nos primeiros anos de sua experiência prática. Além disso, deve-se ter em mente dois fatores: os olhos do médico podem enganar-se no julgamento de benignidade, e achados incidentais de neoplasias malignas em lesões benignas podem acontecer.

Ao se analisar a relação custo/benefício de enviar todo fragmento de pele, sem exceção, para exame anatomopatológico, Lowy, Willis e Abrams ${ }^{3}$ mostraram que houve insignificante aumento no número de lesões consideradas "sérias" pelos autores quando o grupo de médicos generalistas estudado passou a referenciar todo e qualquer fragmento de pele para análise histológica. Os autores compararam dois períodos de tempo - no primeiro, 257 médicos generalistas faziam a seleção do que enviar para exame anatomopatológico; no segundo, eles foram orientados a enviar simplesmente tudo o que fosse retirado da pele de seus pacientes. Embora o número de casos referidos aos serviços de patologia tenha aumentado em $29 \%$, não houve alteração estatisticamente significativa no número de neoplasias malignas enviadas para exame. Alguns artigos mostraram opinião contrária ao estudo daqueles autores, com especulações sobre a acuidade diagnóstica e confiabilidade de se fazer apenas análise macroscópica da lesão de pele, com o risco de se perder o diagnóstico de tumores malignos. ${ }^{4,5,6}$

Ceratoses seborreicas, pólipos fibroepiteliais (acrocórdons), cistos epidérmicos e nevos melanocíticos "comuns" são alguns exemplos de lesões de pele que nem sempre são mandadas para exame anatomopatológico, sendo dispensadas no lixo. Izikson e colaboradores ${ }^{7}$ revisaram 9.204 exames anatomopatológicos nos quais a lesão de pele foi diagnosticada clinicamente como ceratose seborreica e encontraram 61 casos de melanoma. Pela semelhança clínica que pode ocorrer entre melanoma e ceratose seborreica pigmentada, aqueles autores enfatizam a necessidade de exame microscópico de todas as ceratoses seborreicas removidas dos pacientes. Em outro estudo, Cascajo, Reichel e Sanchez ${ }^{8}$ encontraram associação de ceratose seborreica com carcinoma basocelular, doença de Bowen, ceratoacantoma e melanoma. Eads et al., com o intuito de analisar a necessidade de submeter lesões com suspeita clínica de ceratose seborréica a estudo anatomopatológico, relataram o diagnóstico de neoplasia maligna em 6,4\% dos casos. Com relação aos acrocórdons, há um estudo que sugere a desobrigação de analisar microscopicamente essas lesões. ${ }^{10}$ Os autores encontraram cinco casos de malignidade em meio a 1.335 lesões com suspeita clínica de pólipo fibroepitelial estudadas. ${ }^{10}$ Por outro lado, raramente schwanoma ${ }^{11}$ e nevo écrino ${ }^{12}$ podem ser confundidos clinicamente com acrocórdons. Os cistos epidérmicos também podem abrigar neoplasias não diagnosticadas clinicamente, como carcinoma basocelular ${ }^{13,14}$ e carcinoma epidermóide. ${ }^{15}$ Finalmente, a utilidade de se avaliar histologicamente todas as lesões com diagnóstico clínico de nevo melanocítico é discutida em um artigo de Reeck e cols. ${ }^{16}$ Os autores citam a posição da Academia Americana de Dermatologia que diz "mesmo clínicos experientes não são capazes de diagnosticar corretamente lesões pigmentadas, algumas vezes, baseando-se apenas em critérios clínicos; portanto, sempre que houver dúvida, biópsia com o estudo anatomopatológico é necessária”. Eles revisaram 7.734 laudos anatomopatológicos de um serviço de dermatopatologia (recebidos em período de 40 dias do ano de 1995) e separaram todos os casos em que o clínico ou cirurgião suspeitava de algum tipo de 
nevo e fazia ou não o diagnóstico diferencial com melanoma. Os autores descobriram que 1,6\% das lesões que não tinham qualquer suspeita clínica de malignidade foram diagnosticadas como melanoma. Já 2,3\% das lesões cujo diagnóstico clínico de nevo tinha algum modificador como "atípico", "irritado", "que cresceu", etc., ou quando o médico solicitava para "excluir malignidade" foram diagnosticadas como neoplasias malignas por estudo histológico, incluindo 12 melanomas, 30 carcinomas basocelulares e três carcinomas epidermóides. Os pesquisadores sugerem que um estudo como esse deve ser considerado ao se tomar uma posição em relação à obrigatoriedade de biópsia com estudo anatomopatológico de lesões melanocíticas.

\section{PARA QUÊ?}

A indicação mais tradicional da realização da biópsia de pele com estudo anatomopatológico é para esclarecimento diagnóstico. ${ }^{17}$ Doenças inflamatórias de pele com diagnóstico clínico duvidoso e tumores ou lesões cutâneas com suspeita de malignidade necessitam desse estudo para definição de tratamento e conduta. Outro uso interessante do método é para confirmação do diagnóstico clínico firmado. O exame anatomopatológico muitas vezes oferece o aval de que o dermatologista necessita para tomar certas decisões terapêuticas. Inegável também é o efeito psicológico suscitado no paciente (e às vezes também no dermatologista) quando tem o diagnóstico clínico ratificado por um exame, sobretudo quando a resposta ao tratamento escolhido não é perfeita. ${ }^{17} \mathrm{O}$ laudo anatomopatológico pode ser requisitado para autorização do uso de certos medicamentos no Sistema Único de Saúde. Por último, a biópsia de pele com estudo anatomopatológico pode ser utilizada para efeitos de registro diagnóstico no seguimento de pacientes por diversos anos. Especialmente no caso de lesões pigmentadas que sofrem o risco de recidiva, pode ser difícil para o dermatologista lembrar e provar no futuro que sua impressão diagnóstica de benignidade e a escolha da técnica cirúrgica foram acertadas no passado. A requisição de exame anatomopatológico e o laudo são documentos e podem ser usados no tribunal a favor ou contra o médico. ${ }^{17}$

\section{COMO?}

A interdependência da clínica e da patologia no diagnóstico das doenças cutâneas é inquestionável. Parafraseando Miller, ${ }^{18}$ a biópsia de pele transcende o mero ato técnico de retirar um fragmento do tegumento do paciente. $\mathrm{O}$ autor enfatiza a necessidade de médicos não dermatologistas serem treinados também em diagnóstico diferencial clínico de lesões de pele e na interpretação dos resultados da análise ana- tomopatológica. Ou seja, fazer a biópsia de pele em si, o lado técnico do procedimento, não é o cerne da questão quando está em pauta sua utilidade prática: diagnóstico anatomopatológico correto. ${ }^{19}$ Exatamente por esse motivo, não há dúvida de que o profissional mais capacitado para fazer uma biópsia de pele adequada é o dermatologista. Sellheyer e Bergfeld ${ }^{20} \mathrm{com}$ pararam a acurácia diagnóstica de lesões cutâneas entre dermatologistas e não dermatologistas. Eles concluíram que os dermatologistas têm capacidade duas vezes maior do que os outros médicos de obter a correlação clinicopatológica acertada. García-Solano et al $^{21}$ mostraram que os diagnósticos histopatológicos realizados nas biópsias de pele referidas por um serviço de dermatologia são mais específicos (77\%) do que os referidos por serviços não dermatológicos (41\%). Isso mostra a importância direta do papel do dermatologista no resultado da biópsia de pele. Escolha da técnica e local da biópsia, informações clínicas pertinentes e hipóteses diagnósticas influenciaram o grau de acurácia do diagnóstico microscópico, segundo aqueles autores. Por outro lado, a experiência do patologista que faz a análise microscópica também colabora para a exatidão do diagnóstico da doença de pele. ${ }^{21,22,23}$

Como o universo das dificuldades diagnósticas em dermatopatologia não é o foco do presente artigo, discute-se a seguir a primeira parte de "como" o dermatologista pode melhorar os resultados anatomopatológicos obtidos ao realizar uma biópsia de pele, iniciando com o preenchimento da requisição do exame anatomopatológico.

Informações clínicas. Todo texto que lida com a relação lesão de pele/exame anatomopatológico enfatiza o grande peso das informações clínicas na conclusão diagnóstica. ${ }^{17-25}$ Idealmente, dados como sexo e idade do paciente, local e técnica de biópsia, duração da doença e/ou da lesão da qual se fez a biópsia, distribuição e configuração das lesões, aspectos morfológicos individuais das lesões, seus sintomas, história do uso de medicamentos e suspeita diagnóstica e/ou diagnósticos diferenciais devem sempre constar da requisição do exame anatomopatológico. Na verdade, a quantidade de informação nunca é exagerada, mas o contrário, informação insuficiente, pode prejudicar enormemente a interpretação dos achados histológicos pelo patologista. No caso específico de doenças inflamatórias cutâneas, nunca é demais lembrar que elas se manifestam com padrões histológicos comuns a mais de uma doença. ${ }^{17,19}$ Enfermidades tão díspares clinicamente como doença de Grover e doença de Darier, por exemplo, são idênticas sob análise microscópica. O quadro 1 mostra três padrões histológicos comuns e as respectivas doenças que fazem parte de cada grupo. Informações clínicas básicas podem evitar 
QuAdro 1: Exemplos de doenças com quadros clínicos diferentes e padrões histológicos semelhantes

\begin{tabular}{|c|c|c|}
\hline \multicolumn{3}{|c|}{ PADRÃO HISTOLÓGICO } \\
\hline Espongiótico & Psoriasiforme & Liquenoide \\
\hline Dermatite de contato* & Psoríase & Líquen plano \\
\hline Dermatite desidrótica* & Sífilis secundária & Sífilis secundária \\
\hline Dermatite atópica* & Pitiríase rubra pilar & Ceratose liquenoide benigna \\
\hline Dermatite fotoalérgica* & Pitiríase rósea (placa "mãe") & $\begin{array}{l}\text { Púrpura pigmentar crônica } \\
\text { (tipo Gougerot-Bloom) }\end{array}$ \\
\hline Dermatite numular* & Nevil** & Lúpus eritematoso \\
\hline Dermatite seborréica* & Dermatite seborreica & Pitiríase liquenoide \\
\hline Micose fungóide & Micose fungoide & Micose fungoide \\
\hline Tinha & Tinha & Dermatite de contato liquenoide \\
\hline Líquen simples crônico & Líquen simples crônico & Erupção medicamentosa \\
\hline Pitiríase rósea & Acrodermatite enteropática & Líquen estriado \\
\hline Eritema anular centrífugo & Pelagra & \\
\hline
\end{tabular}

* Doença considerada do "grupo eczema"; ** Nevo epidérmico verrucoso inflamatório linear

o constrangimento de um patologista diagnosticar líquen plano no caso de uma ceratose liquenóide benigna, por exemplo.

\section{CONCLUSÃO}

O diagnóstico histológico de uma lesão de pele só não é considerado definitivo em raríssimas situações, e o laudo anatomopatológico tem peso de documento médico e legal. O médico quando retira um fragmento de pele do paciente pode ou não desperdiçar a chance de ter o diagnóstico estabelecido por um exame padrão-ouro. A biópsia de pele com estudo anatomopatológico é indicada para qualquer situação de dúvida no julgamento clínico, quer seja diagnóstica, quer seja terapêutica. O laudo anatomopatológico não só esclarece ou confirma o diagnóstico e auxilia na conduta clínica ou cirúrgica, mas pode ser fator determinante na relação médico/paciente, com possíveis efeitos psicológicos em ambas as partes, aumentando a certeza do médico em seu diagnóstico clínico e a confiança do paciente na conduta do médico.

\section{AGRADECIMENTOS:}

Ao dr. José Fillus Neto, dermatopatologista, e dr. Sergio Z. Serafini, dermatologista, meus mestres e modelos na profissão. 


\section{REFERÊNCIAS}

1. Legislação. Pareceres do Conselho Federal de Medicina. [homepage]. [Acesso em 6 de abril de 2009]. Disponível em: http://www.portalmedico.org.br/ pareceres/cfm/1995/44_1995.htm

2. Pareceres da Sociedade Brasileira de Patologia. [homepage]. [Acesso em 6 de abril de 2009]. Disponível em http://www.sbp.org.br/publicacoes/ pareceres.aspx?id $=52$

3. Lowy A, Willis D, Abrams K. Is histological examination of tissue removed by general practitioners always necessary? Before and after comparison of detection rates of serious skin lesions. BMJ. 1997;315(7105):406-8.

4. Parslew R, Rhodes L. Is histological examination of tissue removed by GP's always necessary. Clinically important skin lesions would have been missed with a selective histological approach. BMJ. 1998; 316 (7133):778.

5. Cross P. Is histological examination of tissue removed by GPs always necessary? Even specialists get the clinical diagnosis wrong. BMJ. 1998;316(7133):778.

6. Suvarna SK, Shortland JR, Smith JH. Is histological examination of tissue removed by GP's always necessary. Over 10 days two important lesions were sent to histopathologists in Sheffield. BMJ. 1998; 316 (7133):778-9.

7. Izikson L, Sober AJ, Mihm MC Jr, Zembowicz A. Prevalence of melanoma clinically resembling seborrheic keratosis: analysis of 9204 cases. Arch Dermatol. 2002;138:1562-6.

8. Cascajo CD, Reichel M, Sanchez JL. Malignant neoplasms associated with seborrheic keratoses. An analysis of 54 cases. Am J Dermatopathol. 1996;18:278-82.

9. Eads TJ, Hood AF, Chuang TY, Faust HB, Farmer ER. The diagnostic yield of histologic examination of seborrheic keratoses. Arch Dermatol. 1997;133:1417-20.

10. Eads TJ, Chuang TY, Fabré VC, Farmer ER, Hood AF. The utility of submitting fibroepithelial polyps for histological examination. Arch Dermatol. 1996;132:1459-62.

11. Kim YK, Ahn SK, Lee SH. A case of perineal schwannoma resembling a skin tag. J Dermatol. 1999;26:687-90.

12. Mahdavy M, Smoller BR. Eccrine nevus presenting as a perianal skin tag: a case report and review of the literature. Am J Dermatopathol. 2002;24:361-3.

13. Mehregan DA, al-Sabah HY, Mehregan AH. Basal cell epithelioma arising from epidermoid cyst. J Dermatol Surg Oncol. 1994;20:405-6.

14. Dini M, Innocenti A, Romano GF. Basal cell carcinoma arising from epidermoid cyst: a case report. Dermatol Surg. 2001;27:585-6.

15. López-Ríos F, Rodríguez-Peralto JL, Castaño E, Benito A.
Squamous cell carcinoma arising in a cutaneous epidermal cyst: case report and literature review. Am J Dermatopathol. 1999;21:174-7.

16. Reeck MC, Chuang TY, Eads TJ, Faust HB, Farmer ER, Hood AF. The diagnostic yield in submitting nevi for histologic examination. J Am Acad Dermatol. 1999; 40:567-71.

17. Scope A, Halpern AC. Diagnostic procedures and devices. In: Wolff K, Goldsmith LA, Katz SI, Gilchrest BA, Paller AS, Leffell DJ, editors. Fitzpatrick's Dermatology in General Medicine. New York: McGraw-Hill; 2007. p. 42.

18. Miller JJ. A biopsy is more than a biopsy. J Gen Intern Med. 1998; 13:62-3.

19. Ackerman AB, Böer A, Bennin B, Gottlieb G. Proper biopsy. In: Histologic Diagnosis of Inflammatory Skin Diseases. An Algorithmic Method Based on Pattern Analysis. Nova York: Ardor Scribendi; 2005. p. 171-9

20. Sellheyer K, Bergfeld WF. A retrospective biopsy study of the clinical diagnostic accuracy of common skin diseases by different specialties compared with dermatology. J Am Acad Dermatol. 2005;52:823-30.

21. García-Solano J, López-Avila A, Acosta J, PérezGuillermo M. Rentabilidad diagnóstica de la biopsia cutânea en las enfermedades inflamatorias de la piel. Estudio comparativo según el servicio que la realiza. Actas Dermosifiliogr. 2005;96:92-7.

22. Penneys NS. Quality assessment of skin biopsy specimens referred to anonymous consultants. Arch Dermatol. 1996;132:1053-6.

23. Kirsner RS, Federman DG. Interpretation of cutaneous biopsy specimens: choice of pathologist by primary care practitioners. South Med J. 2001;94:461-3.

24. Charlton R. Diagnosis and biopsy specimens. Am J Dermatopathol. 1981;3:234.

25. Boyd AS, Neldner KH. How to submit a specimen for cutaneous pathology analysis. Using the '5 D's' to get the most from biopsies. Arch Fam Med. 1997;6:64-6.

ENDEREÇO PARA CORRESPONDÊNCIA / MAILING ADDRESS:
Betina Werner
Rua. Dr. Nelson de Souza Pinto, 759
82200 O60 Curitiba, PR.
Tel./Fax: 41323219064132323524
email: betina.werner@gmail.com

ENDEREÇO PARA CORRESPONDÊNCIA / MAILING ADDRESS: Betina Werner

Rua. Dr. Nelson de Souza Pinto, 759 82200060 Curitiba, PR. email: betina.werner@gmail.com 